\title{
Racional e Desenho do Registro Brasileiro de Implante de Bioprótese Aórtica por Cateter
}

\author{
Fábio Sândoli de Brito Júnior ${ }^{1}$, Dimytri Siqueira ${ }^{2}$, Rogério Sarmento-Leite ${ }^{3}$, Luiz Antonio Carvalho ${ }^{4}$, \\ Pedro Alves Lemos Neto ${ }^{5}$, José Armando Mangione ${ }^{6}$, Cesar Rocha Medeiros ${ }^{7}$, Paulo Caramori ${ }^{8}$, \\ Marco Wainstein ${ }^{9}$, Alcides Zago ${ }^{10,11}$, José Mariani Junior ${ }^{5}$, Adriano Dias Dourado Oliveira ${ }^{12}$, \\ Luiz Eduardo São Thiago ${ }^{13}$, Márcio Andrade ${ }^{14}$, Alexandre Abizaid ${ }^{2}$, Marco A. Perin', \\ Eberhard Grube ${ }^{15}$, J. Eduardo Sousa ${ }^{2,16}$
}

\section{RESUMO}

Introdução: A substituição cirúrgica da valva aórtica é o tratamento de eleição para pacientes com estenose aórtica acentuada. Entretanto, o risco cirúrgico aumenta expressivamente com o avançar da idade e com a associação de comorbidades. Recentemente, iniciou-se a experiência com o implante por cateter de biopróteses aórticas no Brasil. Justifica-se, portanto, a criação de um registro nacional para conhecimento dos resultados desse tipo de procedimento em nosso meio. Descrevemos o racional e o desenho do Registro Brasileiro de Implante de Bioprótese Aórtica por Cateter. Métodos: Trata-se de um registro multicêntrico, prospectivo, que incluirá 200 pacientes portadores de estenose valvar aórtica sintomática de alto risco cirúrgico submetidos a implante por cateter de bioprótese valvular aórtica em centros brasileiros. O desfecho primário do estudo é a mortalidade por qualquer causa aos 30 dias, 12 meses e 24 meses. Os desfechos secundários avaliados incluem, entre outros: 1) sucesso técnico do implante da bioprótese; 2) mortalidade cardiovascular aos 30 dias, 12 meses e 24 meses; 3) desfecho combinado de segurança aos 30 dias; e 4) desfecho combinado de eficácia aos 12 meses e 24 meses. Conclusões: A análise dos resultados desse registro

\footnotetext{
${ }^{1}$ Hospital Albert Einstein - São Paulo, SP, Brasil.

2 Instituto Dante Pazzanese de Cardiologia - São Paulo, SP, Brasil.

${ }^{3}$ Instituto de Cardiologia do Rio Grande do Sul - Fundação Universitária de Cardiologia (IC-FUC) - Porto Alegre, RS, Brasil.

${ }^{4}$ Hospital Pró-Cardíaco - Rio de Janeiro, RJ, Brasil.

${ }^{5}$ Hospital Sírio Libanês - São Paulo, SP, Brasil.

${ }^{6}$ Hospital Beneficência Portuguesa de São Paulo - São Paulo, SP, Brasil.

${ }^{7}$ Hospital Copa D'Or - Rio de Janeiro, RJ, Brasil.

${ }^{8}$ Hospital São Lucas - Pontifícia Universidade Católica do Rio Grande do Sul

- Porto Alegre, RS, Brasil.

${ }^{9}$ Associação Hospitalar Moinhos de Vento - Porto Alegre, RS, Brasil.

${ }^{10}$ Hospital Mãe de Deus - Porto Alegre, RS, Brasil.

11 Hospital de Clínicas de Porto Alegre - Porto Alegre, RS, Brasil.

${ }^{12}$ Hospital Santa Izabel - Santa Casa de Misericórdia da Bahia - Salvador, BA, Brasil.

${ }^{13}$ SOS Cardio - Florianópolis, SC, Brasil.

${ }^{14}$ Hospital Central do Exército - Rio de Janeiro, RJ, Brasil.

${ }^{15}$ Hospital Alemão Oswaldo Cruz - São Paulo, SP, Brasil.

${ }^{16}$ Hospital do Coração - Associação do Sanatório Sírio - São Paulo, SP, Brasil. Correspondência: Fábio Sândoli de Brito Júnior. Rua Dom Armando Lombardi, 819/82-A - São Paulo, SP, Brasil - CEP 05616-011

E-mail: fsbrito@einstein.br

Recebido em: 1\%/6/2011 • Aceito em: 5/6/2011
}

\section{ABSTRACT}

Rationale and Design of the Brazilian Transcatheter Aortic Valve Implantation Registry

Background: Surgical replacement of the aortic valve is the therapy of choice for patients with severe aortic stenosis. However, the surgical risk increases dramatically as age advances and with the association of comorbidities. Recently, transcatheter aortic valve implantation has been introduced in Brazil. Therefore, it was important to create a national registry to determine the outcomes of this procedure in our country. The rationale and design of the Brazilian Transcatheter Aortic Valve Implantation Registry is described. Methods: This is a multicenter prospective registry, including 200 patients with symptomatic aortic valve stenosis and high surgical risk submitted to transcatheter aortic valve implantation in Brazilian institutions. The primary endpoint of the study is all cause mortality at 30 days, 12 and 24 months. Secondary endpoints, among others, are: 1) technical success of transcatheter valve implantation; 2) cardiovascular mortality at 30 days, 12 and 24 months; 3) composite safety endpoint at 30 days; and 4) composite efficacy endpoint at 12 and 24 months. Conclusions: The 
nacional permitirá avaliar os defechos do implante percutâneo de biopróteses aórticas em nosso meio.

DESCRITORES: Estenose da valva aórtica. Implante de prótese de valva cardíaca. Bioprótese. Cateteres.

A substituição cirúrgica da valva aórtica é, há décadas, o tratamento de eleição para pacientes com estenose aórtica acentuada, determinando alívio dos sintomas e aumento da sobrevida. Entretanto, o risco cirúrgico aumenta expressivamente com o avançar da idade e com a associação de comorbidades, o que faz com que mais de um terço dos octogenários com estenose aórtica sintomática seja recusado para a cirurgia. ${ }^{1,2}$ Nesses pacientes, a valvoplastia aórtica com cateter-balão permite melhora apenas temporária dos sintomas e do gradiente de pressão transvalvar, em decorrência da alta incidência de reestenose, sendo indicada, atualmente, apenas excepcionalmente, como medida paliativa ou como ponte para o tratamento cirúrgico. ${ }^{3,4}$ Esses achados estimularam o desenvolvimento de dispositivos para a substituição da valva aórtica por cateter. Em 2002, Cribier et al. ${ }^{5}$ realizaram, com sucesso, o primeiro implante percutâneo de uma bioprótese valvular aórtica em seres humanos, dando início a uma nova era na cardiologia intervencionista. Em 2004, Grube et al. ${ }^{6}$ implantaram a primeira prótese valvular aórtica CoreValve ${ }^{\mathrm{TM}}$ (CoreValve ReValving System, Medtronic, Inc., Minneapolis, Estados Unidos). Atualmente cerca de 25 mil pacientes já foram tratados com esse dispositivo no mundo e a experiência acumulada com o emprego dessas biopróteses em pacientes com contraindicação ou com alto risco cirúrgico indica que os resultados são bastante promissores..$^{7-12}$

Recentemente, iniciou-se a experiência com o sistema CoreValve ${ }^{\text {TM }}$ no Brasil, ${ }^{13,14}$ que motivou a criação de um registro nacional para aferir os resultados do implante percutâneo de biopróteses aórticas em nosso meio.

\section{MÉTODOS}

\section{Desenho do registro}

Registro multicêntrico, pós-comercialização, desenhado para coletar dados de pacientes submetidos a implante percutâneo de CoreValve ${ }^{\mathrm{TM}}$. Trata-se de um registro aberto, que incluirá 200 pacientes consecutivos, tratados em centros brasileiros que realizaram ao menos três procedimentos, a partir de janeiro de 2008.

\section{Objetivo}

O objetivo deste estudo é avaliar os resultados do procedimento e a evolução tanto intra-hospitalar como tardia de pacientes submetidos a implante percutâneo de bioprótese aórtica. analysis of the results of this national registry will enable us to evaluate the outcomes of transcatheter aortic valve implantation in our country.

KEY-WORDS: Aortic valve stenosis. Heart valve prosthesis implantation. Bioprosthesis. Catheters.

\section{Seleção de pacientes}

Atualmente, a indicação do implante percutâneo de biopróteses aórticas restringe-se a um seleto grupo de pacientes, que, pela idade avançada ou por comorbidades, têm contraindicação ou risco elevado para o tratamento cirúrgico convencional. Nesse grupo, a abordagem por cateter tem elevada chance de sucesso e pode oferecer mortalidade inferior àquela esperada com o tratamento cirúrgico. $7,10-12,15$

O risco cirúrgico pode ser estimado por diversos escores. Os mais empregados são o EuroSCORE ${ }^{16}$ e o $\mathrm{STS}^{17}$, validados em centros europeus e americanos, respectivamente. De maneira geral, alguns critérios objetivos podem ser utilizados na definição do paciente de alto risco cirúrgico e, portanto, candidato à substituição por cateter da valva aórtica:

1. idade $\geq 75$ anos e EuroSCORE $\geq 15 \%$ ou STS $\geq 10 \%$; e

2. idade $\geq 65$ anos e pelo menos uma comorbidade associada (cirrose hepática, hipertensão arterial pulmonar, doença pulmonar obstrutiva crônica, aorta em porcelana, radioterapia torácica prévia, embolia pulmonar recorrente, doença do tecido conectivo, caquexia).

A avaliação do risco, em populações de pacientes geriátricos, pode ser obtida também por escores de fragilidade, que levam em consideração critérios clínicos, laboratoriais e funcionais. O escore de fragilidade de Columbia, empregado no estudo randomizado Placement of Aortic Transcatheter Valves (PARTNER) ${ }^{18}$, é um dos escores utilizados em estudos que avaliam o implante percutâneo valvular aórtico. Após a seleção do candidato pelos critérios clínicos, faz-se a análise de parâmetros morfológicos do complexo aórtico e da via de acesso, objetivando determinar a exequibilidade técnica da substituição valvar por cateter. ${ }^{19}$ Para isso, o ecocardiograma, a angiotomografia de múltiplos detectores, a aortografia e a arteriografia do território ilíacofemoral são úteis e fornecem as informações essenciais para o planejamento do procedimento. A coronariografia também é útil para descartar a presença de doença arterial coronária grave associada.

\section{O sistema CoreValve ${ }^{\mathrm{TM}}$}

A bioprótese CoreValve ${ }^{T M}$ é, até o presente momento, a única aprovada pela Agência Nacional de Vigilância Sanitária (Anvisa) para uso clínico no Brasil. Esse dispo- 
sitivo, em sua terceira geração, consiste de três folhetos de pericárdio porcino, montados e suturados em um stent de nitinol de $5 \mathrm{~cm}$ de comprimento, autoexpansível. A parte inferior do stent tem grande força radial para afastar lateralmente os folhetos calcificados da valva aórtica nativa, evitar a retração elástica, minimizar a regurgitação perivalvar e fixar adequadamente a prótese na via de saída do ventrículo esquerdo. A parte média do stent é onde se encontram suturados os folhetos, tendo menor diâmetro para evitar o comprometimento dos óstios coronários. A parte superior do stent se expande para fixação na aorta ascendente e alinhamento da endoprótese. A malha do stent é suficientemente aberta para permitir o acesso de cateteres aos óstios coronários, depois de implantada a bioprótese. Para o implante, o stent e os folhetos da prótese são contraídos no interior de uma bainha de $18 \mathrm{~F}(6 \mathrm{~mm})$ de diâmetro, permitindo que o procedimento seja realizado por acesso retrógrado. O sistema CoreValve ${ }^{T M}$, atualmente, encontra-se disponível nos tamanhos de $26 \mathrm{~mm}$ e $29 \mathrm{~mm}$, para emprego em pacientes com anel valvar de $20 \mathrm{~mm}$ a $23 \mathrm{~mm}$ e de $24 \mathrm{~mm}$ a $27 \mathrm{~mm}$, respectivamente.

\section{O procedimento percutâneo}

O preparo para a substituição por cateter da valva aórtica consiste na administração de aspirina (100 mg) e clopidogrel (dose de ataque de $300 \mathrm{mg}$, seguida de 75 mg por dia), com início na véspera do procedimento, e manutenção por seis meses. Recomenda-se antibioticoprofilaxia com cefalosporinas de primeira geração. Em geral, a intervenção por cateter pode ser realizada com anestesia local e sedação leve, mas, dependendo da condição clínica do paciente, a anestesia geral pode ser indicada. $\mathrm{O}$ acesso retrógrado pode ser obtido por punção ou dissecção da artéria femoral ou por dissecção da artéria subclávia. A seguir, faz-se a valvoplastia aórtica com balão, como pré-dilatação, e libera-se a endoprótese valvular. Durante a pré-dilatação por balão, utiliza-se marca-passo provisório para elevar a frequência cardíaca até 200 bpm a 220 bpm, evitando o deslocamento do balão no momento de sua insuflação. Após o implante da endoprótese, o marca-passo também é útil para a manutenção do ritmo cardíaco no caso de ocorrerem bradiarritmias e bloqueio atrioventricular total, devendo ser mantido por, pelo menos, 24 horas a 48 horas após a substituição por cateter da valva aórtica. A realização de aortografia ao final da intervenção é útil para avaliar a presença de regurgitação perivalvar.

\section{Resultados do implante por cateter de biopróteses aórticas}

Estudos clínicos recentes demonstraram a exequibilidade, a segurança e a eficácia desse tipo de intervenção, com resultados que podem ser resumidos da seguinte forma:

1. O sucesso do procedimento está estritamente relacionado à experiência, sendo o índice de sucesso de aproximadamente $90 \%$ sistematicamente relatado após a superação da curva de aprendizado. A embolização da prótese durante o procedimento ocorre raramente, em cerca de $1 \%$ dos casos.

2. A mortalidade aos 30 dias varia, nas publicações, de $5 \%$ a $18 \%$, dependendo da complexidade tanto clínica como anatômica dos casos tratados. Deve-se ressaltar, entretanto, que essas taxas de mortalidade são muito inferiores àquelas estimadas pelo EuroSCORE para o tratamento cirúrgico desses mesmos pacientes. Algumas publicações apresentam seguimento clínico de até dois anos, com sobrevida de $70 \%$ a $80 \%$ e melhora expressiva da condição clínica da maior parte dos pacientes. A maioria das mortes nesse período decorre de comorbidades e não se relaciona a problemas com a prótese.

3. Os estudos divulgados são unânimes em demonstrar o adequado funcionamento das biopróteses aórticas implantadas por cateteres. Após o implante, atinge-se área valvar de aproximadamente $1,5 \mathrm{~cm}^{2}$ e nota-se queda importante no gradiente de pressão transvalvar aórtico. Essa melhora hemodinâmica precoce se reflete, rapidamente, na melhora dos sintomas de insuficiência cardíaca congestiva dos pacientes tratados. A médio prazo, existe melhora da função e redução da hipertrofia do ventrículo esquerdo. Aproximadamente $50 \%$ a $70 \%$ dos casos apresentam regurgitação perivalvar de pequena ou moderada intensidade ao final da intervenção, que tende a diminuir durante o seguimento. Regurgitação perivalvar acentuada ocorre em torno de $5 \%$ dos casos e pode ser manuseada com redilatações da prótese com balões ou até com o implante de uma prótese adicional. Não há relato de degeneração estrutural da prótese no seguimento de até dois anos.

4. Outras complicações relacionadas ao procedimento são descritas nesta população de alto risco e repleta de comorbidades. Complicações vasculares na via de acesso ocorrem em 1,9\% a 15\% dos casos. Há relato da ocorrência de acidente vascular cerebral em $1,9 \%$ a $10 \%$ dos casos. A oclusão de artérias coronárias pelas biopróteses é muito rara $(<1 \%)$. Bloqueios atrioventriculares com necessidade de implante de marca-passo permanente são detectados em até $20 \%$ a $30 \%$ dos pacientes submetidos a substituição por cateter da valva aórtica com o dispositivo CoreValve ${ }^{\mathrm{TM}}$ e em cerca de $5 \%$ daqueles tratados com a bioprótese Edwards-Sapien. Cabe destacar que esse tipo de problema também pode ocorrer após o tratamento cirúrgico da estenose aórtica. ${ }^{20}$

\section{Desfechos}

\section{Desfecho primário:}

- Mortalidade por qualquer causa aos 30 dias, 12 meses e 24 meses do procedimento.

\section{Desfechos secundários:}

- Taxa de sucesso técnico do implante da bioprótese valvular.

- Taxa de mortalidade cardiovascular aos 30 dias, 12 meses e 24 meses. 
- Desfecho combinado de segurança aos 30 dias: morte por qualquer causa, acidente vascular encefálico maior, sangramento com risco de morte ou incapacitante, insuficiência renal aguda estágio 3, infarto periprocedimento ou novo procedimento percutâneo ou cirúrgico para correção de disfunção valvar.

- Desfecho combinado de eficácia aos 12 meses e 24 meses: morte por qualquer causa ocorrendo 30 dias após o procedimento, internação hospitalar por descompensação cardíaca relacionada à valva aórtica, disfunção da bioprótese (estenose: área valvar aórtica $<1,2 \mathrm{~cm}^{2}$ e gradiente transvalvar aórtico $>20 \mathrm{mmHg}$ ou velocidade de pico $>3 \mathrm{~m} / \mathrm{s}$, ou insuficiência aórtica moderada ou acentuada).

\section{Outros desfechos:}

- Evolução da classe funcional de insuficiência cardíaca (classificação da New York Heart Association, de I a IV) no seguimento clínico de 6 meses, 12 meses e 24 meses.

- Taxas de infarto periprocedimento (72 horas) e espontâneo aos 30 dias, 12 meses e 24 meses.

- Taxa de complicações vasculares durante os primeiros 30 dias.

- Taxa de acidente vascular encefálico aos 30 dias, 12 meses e 24 meses.

- Taxa de complicações hemorrágicas aos 30 dias, 12 meses e 24 meses.

- Taxa de insuficiência renal aguda aos 30 dias.

- Desfechos avaliados por ecocardiogramas (pré-intervenção e pós-intervenção, na fase hospitalar, após 6-12 meses e após 18-24 meses): gradiente de pressão transvalvar aórtico; função ventricular esquerda; grau de regurgitação valvar ou perivalvar aórtica; e grau de insuficiência mitral.

- Taxa de complicações relacionadas à bioprótese, incluindo novos distúrbios de condução e implante de marca-passo permanente.

\section{Definições}

Todos os desfechos do estudo seguem os critérios estabelecidos pelo Valve Academic Research Consortium Consensus on Event Definition ${ }^{21}$, divulgado no congresso EuroPCR 2010, em Paris (França).

\section{Mortalidade cardiovascular:}

a) Morte por causa cardíaca (infarto, tamponamento cardíaco, insuficiência cardíaca, arritmia, etc.).

b) Morte súbita ou de causa indeterminada.

c) Morte relacionada ao procedimento, incluindo as mortes relacionadas a complicações ou a tratamento de complicações do procedimento.

d) Morte causada por doença vascular não-coronária (tromboembolismo pulmonar, aneurisma/dissecção de aorta, acidente vascular cerebral ou óbito por outra causa vascular). e) Todos os outros óbitos são classificados como não-cardiovasculares, incluindo infecção, câncer, suicídio, trauma, etc.

\section{Infarto do miocárdio periprocedimento ( $<72$ horas)}

Após implante da bioprótese, considerar-se-á infarto do miocárdio quando houver elevação dos níveis de creatina quinase fração $M B(C K-M B)$ na vigência de pelo menos um dos seguintes itens:

a) Sinais ou sintomas sugestivos de isquemia miocárdica.

b) Alterações eletrocardiográficas.

c) Exames de imagem sugestivos de isquemia miocárdica, como déficit de contração segmentar na avaliação pelo ecocardiograma.

As elevações de CK-MB só serão consideradas significativas para o diagnóstico de infarto caso se identifique, em duas amostras consecutivas colhidas após o procedimento (intervalo $>6$ horas entre as duas amostras), elevação $\geq 20 \%$ na segunda amostra e valor de pico > 10 vezes o limite superior da normalidade. Também serão consideradas para o diagnóstico de infarto as elevações de CK-MB > 5 vezes o limite superior da normalidade, desde que associadas ao surgimento de novas ondas $Q$ patológicas em duas ou mais derivações contíguas.

\section{Infarto do miocárdio espontâneo (> 72 horas)}

Considerar-se-á infarto do miocárdio espontâneo quando houver elevação de marcadores cardíacos (preferencialmente troponina), com pelo menos um dos seguintes achados:

a) ECG demonstrando surgimento de novas alterações de ST-T ou de novo bloqueio do ramo esquerdo ou surgimento de novas ondas Q patológicas em duas ou mais derivações contíguas.

b) Exames de imagem demonstrando nova perda de miocárdio viável ou alteração de contratilidade.

c) Morte cardíaca inesperada com um dos seguintes achados: parada cardíaca com novo bloqueio de ramo esquerdo ou elevação do segmento ST; evidência de trombo na angiografia coronária; achado de autopsia, mesmo que sem elevação de marcadores cardíacos documentada.

\section{Acidente vascular cerebral}

Surgimento abrupto de déficit neurológico focal ou global, sem alterações metabólicas, presença de medicamentos ou quadro infeccioso que justifiquem o quadro, associado a um dos seguintes achados:

a) Duração $\geq 24$ horas ou, se $<24$ horas, associado à realização de alguma intervenção terapêutica (por exemplo, trombólise, angioplastia intracraniana).

b) Diagnóstico confirmado por neurologista, por método de imagem (tomografia computadorizada, resso- 
nância magnética, angiografia) ou por punção lombar com líquor hemorrágico característico de hemorragia intracraniana.

c) Déficit neurológico resultando em óbito.

O acidente vascular encefálico será classificado em maior (escore de Rankin modificado $\geq 2$ ) ou menor (escore de Rankin modificado < 2), de acordo com a avaliação clínica. ${ }^{22,23}$ Considerar-se-á ataque isquêmico transitório quando houver surgimento abrupto de déficit neurológico focal de resolução rápida $(<24$ horas) e com exames de imagem sem lesão cerebral.

\section{Complicações hemorrágicas}

Serão classificadas em três categorias, dependendo da gravidade do quadro:

a) Hemorragia com risco de morte ou incapacitante:

- sangramento em área ou órgão críticos (por exemplo, intracraniano, pericárdico com necessidade de pericardiocentese);

- sangramento causando choque hipovolêmico ou com necessidade de tratamento cirúrgico;

- sangramento de causa aparente com queda da hemoglobina $\geq 5 \mathrm{~g} / \mathrm{dl}$ ou necessidade de transfusão de $\geq 4$ unidades de concentrado de hemácias ou sangue total.

b) Sangramento maior:

- sangramento de causa aparente com queda da hemoglobina $\geq 3 \mathrm{~g} / \mathrm{dl}$ ou necessidade de transfusão de 2-3 unidades de concentrado de hemácias ou sangue total.

c) Sangramento menor:

- qualquer sangramento que não se qualifica como com risco de morte ou maior (por exemplo, hematoma no sítio de acesso).

\section{Insuficiência renal aguda}

Classificada em três estágios, de acordo com os critérios de RIFLE ${ }^{24}$ modificados:

a) Estágio 1: aumento da creatinina sérica de 1,5 vez a 2 vezes em relação ao valor basal ou aumento $>0,3 \mathrm{mg} / \mathrm{dl}$ nas primeiras 72 horas após o procedimento.

b) Estágio 2: aumento da creatinina sérica de 2 a 3 vezes em relação ao valor basal nas primeiras 72 horas após o procedimento.

c) Estágio 3: aumento da creatinina sérica $>3$ vezes em relação ao valor basal ou creatinina sérica $\geq 4 \mathrm{mg} / \mathrm{dl}$ com aumento de pelo menos 0,5 mg/dl nas primeiras 72 horas após o procedimento ou necessidade de diálise.

\section{Complicações vasculares}

\section{a) Maior:}

- dissecção de aorta torácica;

- complicação no sítio de acesso causando morte ou necessidade de transfusão de $\geq 4$ unidades de concentrado de hemácias ou sangue total ou necessidade de intervenção cirúrgica ou por cateter ou resultando em lesão irreversível em algum órgão;

- embolização distal (exceto território cerebral) de origem vascular necessitando de cirurgia ou resultando em amputação ou lesão irreversível em algum órgão.

b) Menor:

- complicação no sítio de acesso com necessidade de transfusão de 2 ou 3 unidades de concentrado de hemácias ou sangue total sem necessidade de intervenção cirúrgica ou por cateter, não resultando em lesão irreversível em algum órgão;

- embolização distal (exceto território cerebral) de origem vascular tratada por embolectomia e/ou trombectomia, não resultando em amputação ou lesão irreversível de órgãos;

- falência de dispositivos hemostáticos resultando em intervenção percutânea (por exemplo, stent-graft) ou cirúrgica e não associada a morte, necessidade de transfusão significativa ( $\geq 4$ unidades) ou lesão irreversível de órgãos.

\section{Disfunção da bioprótese}

a) Estenose da bioprótese aórtica significante: critérios definidos nas diretrizes da American Society of Echocardiography (Tabela 1). ${ }^{25}$

b) Insuficiência da bioprótese aórtica: critérios definidos nas diretrizes da American Society of Echocardiography (Tabela 2). ${ }^{25}$

\section{Complicações associadas à bioprótese}

a) Complicações por contiguidade:

- novos distúrbios de condução: lesão do nodo atrioventricular/feixe de His determinando distúrbios de condução atrioventricular e/ou bloqueio do ramo esquerdo e/ou necessidade de implante de marca-passo permanente (deve-se registrar o momento da ocorrência dessas alterações, se intraprocedimento, na fase hospitalar ou após a alta);

- obstrução coronária aguda: comprometimento do óstio coronário, embolização, deslocamento dos folhetos nativos, etc.

- nova disfunção valvar mitral;

- nova comunicação interventricular, perfuração ou rotura da raiz da aorta.

b) Endocardite

c) Hemólise

d) Complicações relacionadas com o implante:

- perfuração de ventrículo esquerdo, migração/ embolização da prótese, necessidade de implante de uma segunda bioprótese (valve-in-valve), etc.

\section{Sucesso da bioprótese valvular}

Uma única bioprótese implantada no local correto e com funcionamento adequado. 


\section{Análise estatística}

As variáveis contínuas serão apresentadas como média e desvio padrão. As variáveis categóricas serão apresentadas como frequências (número e porcentagem). Quando análises de subgrupos forem realizadas, serão utilizados os testes de qui-quadrado ou exato de Fisher para a análise de variáveis categóricas; para variáveis contínuas, será utilizado o teste $t$ de Student. Para a análise sequencial das variáveis no mesmo paciente, será utilizada análise de variância (ANOVA) para medidas repetidas. Análises de regressão logística uni e multivariada serão usadas para avaliar preditores de eventos adversos e de outros desfechos secundários. As curvas de sobrevida livre de eventos de Kaplan-Meier serão apresentadas para os desfechos clínicos primários e secundários. Os testes de log-rank e hazard ratio serão empregados para comparações entre curvas atuariais de Kaplan-Meier. Valores de $\mathrm{P}<0,05$ serão considerados estatisticamente significantes.

\section{Termo de consentimento livre e esclarecido}

O consentimento livre e esclarecido deve ser obtido de todos os pacientes, cujos dados serão incorporados ao website do registro.

\section{Considerações éticas}

Cabe ao investigador participante assegurar que este programa seja conduzido em conformidade com a Declaração de Helsinque e com todas as diretrizes aplicáveis do International Conference on Harmonisation $(\mathrm{ICH})$ e de todos os regulamentos locais e nacionais.

TABELA 1

Critérios para o diagnóstico de estenose de bioprótese aórtica significante

\begin{tabular}{|c|c|c|c|}
\hline Parâmetro & $\begin{array}{c}\text { Valor } \\
\text { normal }\end{array}$ & $\begin{array}{c}\text { Possível } \\
\text { estenose aórtica }\end{array}$ & $\begin{array}{l}\text { Estenose aórtica } \\
\text { significante }\end{array}$ \\
\hline Velocidade de pico, m/s & $<3$ & $3-4$ & $>4$ \\
\hline Gradiente médio, mmHg & $<20$ & 20-35 & $>35$ \\
\hline $\begin{array}{l}\text { Relação velocidade da via de saída } \\
\text { do VE/velocidade transvalvar aórtica }\end{array}$ & $\geq 0,30$ & $0,29-0,25$ & $<0,25$ \\
\hline Área efetiva do orifício valvar, $\mathrm{cm}^{2}$ & $>1,2$ & $1,2-0,8$ & $<0,8$ \\
\hline Padrão da curva do jato ao Doppler & $\begin{array}{l}\text { Triangular, } \\
\text { pico precoce }\end{array}$ & $\begin{array}{l}\text { Triangular a } \\
\text { intermediário }\end{array}$ & $\begin{array}{l}\text { Arredondada, } \\
\text { contorno simétrico }\end{array}$ \\
\hline Tempo de aceleração, ms & $<80$ & 80-100 & $>100$ \\
\hline
\end{tabular}

TABELA 2

Critérios para o diagnóstico de insuficiência de bioprótese aórtica significante

\begin{tabular}{|c|c|c|c|}
\hline Parâmetro & $\begin{array}{l}\text { Insuficiência } \\
\text { aórtica leve }\end{array}$ & $\begin{array}{l}\text { Insuficiência } \\
\text { aórtica moderada }\end{array}$ & $\begin{array}{l}\text { Insuficiência } \\
\text { aórtica grave }\end{array}$ \\
\hline $\begin{array}{l}\text { Estrutura e mobilidade da } \\
\text { prótese mecânica ou biológica }\end{array}$ & Usualmente normal & Anormal & Anormal \\
\hline Tamanho do ventrículo esquerdo & Normal & Normal ou levemente dilatado & Dilatado \\
\hline \multicolumn{4}{|l|}{$\begin{array}{l}\text { Parâmetros do Doppler } \\
\text { (qualitativos ou semiquantitativos) }\end{array}$} \\
\hline $\begin{array}{l}\text { Largura do jato ao Doppler colorido } \\
\text { (\% do diâmetro da via de saída do VE) }\end{array}$ & Estreito ( $\leq 25 \%)$ & Intermediário (26\%-64\%) & Largo $(\geq 65 \%)$ \\
\hline Densidade do jato: Doppler contínuo & Incompleto, fraco & Denso & Denso \\
\hline $\begin{array}{l}\text { Tempo de meia pressão do jato } \\
\text { regurgitante (PHT, ms): Doppler contínuo }\end{array}$ & Lento (> 500) & Intermediário (200-500) & Rápido (< 500) \\
\hline $\begin{array}{l}\text { Fluxo em via de saída do VE vs. } \\
\text { fluxo pulmonar: Doppler pulsado }\end{array}$ & Levemente aumentado & Intermediário & Muito aumentado \\
\hline $\begin{array}{l}\text { Reversão do fluxo diastólico em } \\
\text { aorta descendente: Doppler pulsado }\end{array}$ & Ausente ou inicial & Intermediário & $\begin{array}{l}\text { Proeminente, } \\
\text { holodiastólico }\end{array}$ \\
\hline \multicolumn{4}{|l|}{ Parâmetros quantitativos do Doppler } \\
\hline Volume regurgitante, $\mathrm{ml} /$ batimento & $<30$ & $30-59$ & $>60$ \\
\hline Fração regurgitante, \% & $<30$ & $30-50$ & $>50$ \\
\hline
\end{tabular}

VE = ventrículo esquerdo. 


\section{Coleta de dados}

Todos os investigadores nas instituições clínicas participantes, incluindo médicos, enfermeiras de pesquisa e coordenadores de pesquisa, que aderirem ao protocolo terão as seguintes responsabilidades: concordar em incorporar os dados necessários diretamente no sistema eletrônico, participar ativamente nas visitas de iniciação do centro e/ou de monitoria, responder a todas as perguntas do centro de coordenação dos dados (Sociedade Brasileira de Hemodinâmica e Cardiologia Intervencionista - $\mathrm{SBHCl}$, São Paulo, SP, Brasil), relatar de forma acurada todos os eventos adversos conhecidos, e preencher todos os módulos de dados eletrônicos de maneira adequada e dentro dos prazos. Informações sobre os pacientes serão mantidas em caráter confidencial, e cada paciente será identificado por um número único usado em formulários eletrônicos de relato do caso para finalidades analíticas.

\section{Coordenação do estudo e centro de gerenciamento de dados}

Este estudo será coordenado e gerenciado pela SBHCI.

\section{Captura eletrônica de dados pelo website}

Cada centro participante incorporará seus dados usando o sistema eletrônico de website do Registro. A $\mathrm{SBHCl}$ assegurará a qualidade e a integridade dos dados e entrará em contato com todos os centros participantes para assegurar a exatidão dos dados. Os centros serão instruídos sobre como usar o sistema de website.

\section{Processo de coleta de dados}

Depois que o treinamento apropriado do website for concluído e todas as aprovações relevantes do centro forem obtidas, os médicos participantes e/ou a equipe local responsável darão início, via Internet, à entrada de todos os dados relevantes dos pacientes no website. Os dados serão submetidos eletronicamente a um servidor seguro e armazenados em posição protegida e confidencial.

\section{Confidencialidade dos dados}

A equipe responsável pela entrada dos dados terá acesso apenas à informação confidencial dos pacientes registrados em seu próprio centro. Os dados serão incorporados a formulários eletrônicos de relato do caso através de uma conexão segura de Internet.

\section{Revisão de segurança}

O desfecho primário do estudo (morte por qualquer causa) será submetido a revisão e adjudicação por um Comitê de Eventos independente.

\section{SUPORTE FINANCEIRO}

Este projeto tem suporte financeiro da Sociedade Brasileira de Hemodinâmica e Cardiologia Intervencionista.

\section{REFERÊNCIAS}

1. Bouma BJ, van Den Brink RB, van Der Meulen JH, Verheul HA, Cheriex EC, Hamer HP, et al. To operate or not on elderly patients with aortic stenosis: the decision and its consequences. Heart. 1999;82(2):143-8.

2. Iung B, Cachier A, Baron G, Messika-Zeitoun D, Delahaye $\mathrm{F}$, Tornos $\mathrm{P}$, et al. Decision-making in elderly patients with severe aortic stenosis: why are so many denied surgery? Eur Heart J. 2005;26(24):2714-20.

3. Kuntz RE, Tosteson AN, Berman AD, Goldman L, Gordon PC, Leonard BM, et al. Predictors of event-free survival after balloon aortic valvuloplasty. N Engl J Med. 1991;325(1):17-23.

4. Otto CM, Mickel MC, Kennedy JW, Alderman EL, Bashore TM, Block PC, et al. Three-year outcome after balloon aortic valvuloplasty. Insights into prognosis of valvular aortic stenosis. Circulation. 1994;89(2):642-50.

5. Cribier A, Eltchaninoff $\mathrm{H}$, Bash A, Borenstein N, Tron C, Bauer $\mathrm{F}$, et al. Percutaneous transcatheter implantation of an aortic valve prosthesis for calcific aortic stenosis: first human case description. Circulation. 2002;106(24):3006-8.

6. Grube E, Laborde JC, Zickmann B, Gerckens U, Felderhoff $T$, Sauren B, et al. First report on a human percutaneous transluminal implantation of a self-expanding valve prosthesis for interventional treatment of aortic valve stenosis. Catheter Cardiovasc Interv. 2005;66(4):465-9.

7. Cribier A, Eltchaninoff $H$, Tron C, Bauer F, Agatiello C, Nercolini D, et al. Treatment of calcific aortic stenosis with the percutaneous heart valve: mid-term follow-up from the initial feasibility studies: the French experience. J Am Coll Cardiol. 2006;47(6):1214-23.

8. Cribier A, Eltchaninoff $\mathrm{H}$, Tron C, Bauer F, Agatiello C, Sebagh L, et al. Early experience with percutaneous transcatheter implantation of heart valve prosthesis for the treatment of end-stage inoperable patients with calcific aortic stenosis. J Am Coll Cardiol. 2004;43(4):698-703.

9. Grube E, Laborde JC, Gerckens U, Felderhoff T, Sauren B, Buellesfeld L, et al. Percutaneous implantation of the CoreValve self-expanding valve prosthesis in high-risk patients with aortic valve disease: the Siegburg first-in-man study. Circulation. 2006;114(15):1616-24.

10. Grube E, Schuler G, Buellesfeld L, Gerckens U, Linke A, Wenaweser $\mathrm{P}$, et al. Percutaneous aortic valve replacement for severe aortic stenosis in high-risk patients using the second- and current third-generation self-expanding CoreValve prosthesis: device success and 30-day clinical outcome. J Am Coll Cardiol. 2007;50(1):69-76.

11. Webb JG, Pasupati S, Humphries K, Thompson C, Altwegg $\mathrm{L}$, Moss R, et al. Percutaneous transarterial aortic valve replacement in selected high-risk patients with aortic stenosis. Circulation. 2007;116(7):755-63.

12. Piazza N, Grube E, Gerckens $U$, den Heijer P, Linke A, Luha $\mathrm{O}$, et al. Procedural and 30-day outcomes following transcatheter aortic valve implantation using the third generation (18 Fr) CoreValve ReValving System: results from the multicentre, expanded evaluation registry 1-year following CE mark approval. Eurolntervention. 2008;4(2):242-9.

13. Perin MA, Brito Junior FS, Almeida BO, Pereira MAM, Abizaid A, Tarasoutchi F, et al. Substituição valvar aórtica percutânea para o tratamento da estenose aórtica: experiência inicial no Brasil. Arq Bras Cardiol. 2009;93(3):299-306.

14. Leite RS, Quadros AS, Prates PRL, Zanatta PA, Salgado Filho PA, Grando T, et al. Implante valvular aórtico percutâneo: experiência inicial do Sul do Brasil. Rev Bras Cardiol Invasiva. 2008;16(4):398-405.

15. Rosengart TK, Feldman T, Borger MA, Vassiliades TA Jr, 
Gillinov AM, Hoercher KJ, et al. Percutaneous and minimally invasive valve procedures: a scientific statement from the American Heart Association Council on Cardiovascular Surgery and Anesthesia, Council on Clinical Cardiology, Functional Genomics and Translational Biology Interdisciplinary Working Group, and Quality of Care and Outcomes Research Interdisciplinary Working Group. Circulation. 2008;117(13): $1750-67$.

16. Roques F, Nashef SA, Michel P, EuroSCORE Study Group. Risk factors for early mortality after valve surgery in Europe in the 1990s: lessons from the EuroSCORE pilot program. J Heart Valve Dis. 2001;10(5):572-7; discussion 577-8.

17. Hattler BG, Madia C, Johnson C, Armitage JM, Hardesty RL, Kormos RL, et al. Risk stratification using the Society of Thoracic Surgeons Program. Ann Thorac Surg. 1994;58(5):1348-52.

18. Leon MB, Smith CR, Mack M, Miller DC, Moses JW, Svensson LG, et al. Transcatheter aortic-valve implantation for aortic stenosis in patients who cannot undergo surgery. N Engl J Med. 2010;363(17):1597-607.

19. Piazza N, de Jaegere P, Schultz C, Becker AE, Serruys PW, Anderson RH. Anatomy of the aortic valvar complex and its implications for transcatheter implantation of the aortic valve. Circ Cardiovasc Intervent. 2008;1(1):74-81.

20. Dawkins S, Hobson AR, Kalra PR, Tang AT, Monro JL, Dawkins KD. Permanent pacemaker implantation after isolated aortic valve replacement: incidence, indications, and predictors. Ann Thorac Surg. 2008;85(1):108-12.

21. Leon MB, Piazza N, Nikolsky E, Blackstone EH, Cutlip DE, Kappetein AP, et al. Standardized endpoint definitions for Transcatheter Aortic Valve Implantation clinical trials: a Con- sensus report from the Valve Academic Research Consortium. J Am Coll Cardiol. 2011;57(3):253-69.

22. Bonita R, Beaglehole R. Recovery of motor function after stroke. Stroke. 1988;19(12):1497-500.

23. Rankin J. Cerebral vascular accidents in patients over the age of 60. II. Prognosis. Scott Med J. 1957;2(5):200-15.

24. Bellomo R, Ronco C, Kellum JA, Mehta RL, Palevsky P; Acute Dialysis Quality Initiative Workgroup. Acute renal failure definition, outcome measures, animal models, fluid therapy and information technology needs: the Second International Consensus Conference of the Acute Dialysis Quality Initiative (ADQI) Group. Crit Care. 2004;8(4):R204-12.

25. Zoghbi WA, Chambers JB, Dumesnil JG, Foster E, Gottdiener JS, Grayburn PA, et al. Recommendations for evaluation of prosthetic valves with echocardiography and doppler ultrasound: a report From the American Society of Echocardiography's Guidelines and Standards Committee and the Task Force on Prosthetic Valves, developed in conjunction with the American College of Cardiology Cardiovascular Imaging Committee, Cardiac Imaging Committee of the American Heart Association, the European Association of Echocardiography, a registered branch of the European Society of Cardiology, the Japanese Society of Echocardiography and the Canadian Society of Echocardiography, endorsed by the American College of Cardiology Foundation, American Heart Association, European Association of Echocardiography, a registered branch of the European Society of Cardiology, the Japanese Society of Echocardiography, and Canadian Society of Echocardiography. J Am Soc Echocardiogr. 2009; 22(9):975-1014; quiz 1082-4.

APÊNDICE

Conflito de interesses

\begin{tabular}{|c|c|c|c|c|c|c|c|}
\hline Nome & $\begin{array}{c}\text { Participação } \\
\text { em estudo }\end{array}$ & Palestrante $^{2}$ & Consultor $^{3}$ & $\begin{array}{c}\text { Comitê } \\
\text { normativo }^{4}\end{array}$ & $\begin{array}{l}\text { Auxílio pessoal/ } \\
\text { institucional }^{5}\end{array}$ & $\begin{array}{c}\text { Textos } \\
\text { científicos } \\
\text { patrocinados }^{6}\end{array}$ & $\begin{array}{c}\text { Participação } \\
\text { acionária }^{7}\end{array}$ \\
\hline Adriano Dias Dourado Oliveira & Não & Não & Não & Não & Não & Não & Não \\
\hline Alcides Zago & Não & Não & Não & Não & Não & Não & Não \\
\hline Alexandre Abizaid & Não & Não & Não & Não & Não & Não & Não \\
\hline Cesar Rocha Medeiros & Não & Não & Não & Não & Não & Não & Não \\
\hline Dimytri Siqueira & Não & Não & Não & Não & Não & Não & Não \\
\hline Eberhard Grube & Não & Não & Sim & Não & Não & Não & Não \\
\hline Fábio Sândoli de Brito Júnior & Não & Sim & Não & Não & Não & Não & Não \\
\hline J. Eduardo Sousa & Não & Não & Não & Não & Não & Não & Não \\
\hline José Armando Mangione & Não & Sim & Não & Não & Não & Não & Não \\
\hline José Mariani Junior & Não & Não & Não & Não & Não & Não & Não \\
\hline Luiz Antonio Carvalho & Não & Sim & Não & Não & Não & Não & Não \\
\hline Luiz Eduardo São Thiago & Não & Não & Não & Não & Não & Não & Não \\
\hline Marco A. Perin & Não & Sim & Sim & Não & Não & Não & Não \\
\hline Marco Wainstein & Não & Não & Não & Não & Não & Não & Não \\
\hline Paulo Caramori & Não & Não & Não & Não & Sim & Não & Não \\
\hline Pedro Alves Lemos Neto & Não & Não & Não & Não & Não & Não & Não \\
\hline Rogério Sarmento-Leite & Não & Sim & Não & Não & Sim & Não & Não \\
\hline \multicolumn{8}{|c|}{$\begin{array}{l}1 \text { = Participou de estudos clínicos e/ou experimentais subvencionados pela indústria farmacêutica ou de equipamentos relacionados } \\
\text { ao Registro?; } 2 \text { = Foi palestrante em atividades ou eventos patrocinados pela indústria relacionados ao Registro?; } 3=\text { Foi (é) membro } \\
\text { de conselho consultivo ou diretivo da indústria farmacêutica ou de equipamentos relacionado ao Registro?; } 4=\text { Participou de comitês } \\
\text { normativos de estudos científicos patrocinados pela indústria relacionados ao Registro?; } 5=\text { Recebeu auxílio pessoal ou instituciona } \\
\text { da indústria relacionado ao Registro?; } 6 \text { = Elaborou textos científicos em periódicos patrocinados pela indústria relacionados ao } \\
\text { Registro?; } 7 \text { = Tem ações da indústria relacionadas ao Registro?. }\end{array}$} \\
\hline
\end{tabular}

